Relations industrielles

Industrial Relations

\title{
Objectives and Standards : An Approach to Planning and Control, by Ernest C. Miller, American Management Association, Inc., New York, 1966, 120 pages.
}

\section{Paul Bélanger}

\section{Volume 22, numéro 2, 1967}

URI : https://id.erudit.org/iderudit/027803ar

DOI : https://doi.org/10.7202/027803ar

Aller au sommaire du numéro

Éditeur(s)

Département des relations industrielles de l'Université Laval

ISSN

0034-379X (imprimé)

1703-8138 (numérique)

Découvrir la revue

Citer ce compte rendu

Bélanger, P. (1967). Compte rendu de [Objectives and Standards : An Approach to Planning and Control, by Ernest C. Miller, American Management

Association, Inc., New York, 1966, 120 pages.] Relations industrielles / Industrial Relations, 22(2), 308-309. https://doi.org/10.7202/027803ar

Tous droits réservés @ C Département des relations industrielles de l'Université Laval, 1967
Ce document est protégé par la loi sur le droit d'auteur. L'utilisation des services d’Érudit (y compris la reproduction) est assujettie à sa politique d'utilisation que vous pouvez consulter en ligne.

https://apropos.erudit.org/fr/usagers/politique-dutilisation/ 
Depuis quatre ans, le département américain du travail effectue des recherches sur la main-d'oeuvre en vertu du "Manpower Development and Training Act» de 1962.

Ce programme de recherches se propose d'atteindre trois objectifs:

1) I'cmélioration ou le perfectionnement des programmes du département;

2) le développement de nouvelles perspectives et de nouvelles solutions aux problèmes de main-d'oeurre ;

3) le dépistage hâtif des problèmes susceptibles de survenir.

Avec de tels objectifs en tête, les chercheurs ont dû toucher à l'ensemble des aspects d'une situation donnée, soit les aspects économiques, sociaux, culturels et éducationnels.

Depuis les débuts, les recherches ont mis l'emphase sur plusieurs problèmes concernont lo main-d'oeurre et notamment: l'impact de l'automation sur l'emploi, le taux élevé de sous-emploi, la forte augmentation de jeunes travailleurs aptes à s'inscrire sur le marché du travail et l'émigration des travailleurs ruraux vers les centres urbains. On peut déceler également un intérêt très marqué à la formation professionnelle des trovailleurs et spécialement aux travailleurs plus âgés et aux handicapés.

Afin d'élargir le plus possible les cadres de son programme, le département utilise des recherchistes apportenant à diverses disciplines et venant des différentes universités, agences gouvernementales et autres organisations variées à la recherche sur les problèmes du monde du travail.

Le rapport, dont il est question ici, trace dans une première partie la liste des projets pour lesquels des contrats ont été accordés durant l'année fiscale 1966. La deuxième partie couvre les contrats qui ont été accordés en 1966 mais qui avaient été conçus avant le premier juillet 1965. La troisième partie contient une description des octrois qui ont été accordés par la « Manpower Research Institutional Grants ». Enfin, les parties 4 et 5 sont consacrées à la description de thèses sur des problèmes dont le thème est la main d'oeuvre et de projets divers dans le domaine de la main-d'oeuvre.

Les résultats de ces recherches ont été utilisés dans l'effort de planification du gouvernement américcin et dans l'édification de certaines politiques. Un certain nombre de projets, qu'on peut retrouver dans la sixième partie du rapport, ont donné naiss-nce à la publication de livres et de monogrophies.

On peut dire, à la rigueur, qu'il s'agit $d^{\prime} u n$ index bibliogrophique s'intéressant assez exhaustivement à la recherche et cuux projets amorcés à partir du MDTA. A ce titre, nous le considérons comme très volable et éminemment susceptible de servir de: volume de références.

\section{Paul BOUCHARD}

\section{Objectives and Standards: An Approach to}

Planning and Control, by Ernest C. Miller, American Management Association, Inc., New York, 1956, 120 pages.

Cette étude est en quelque sorte la continuation et la confirmation d'un ouvrage publié en 1960 par la même maison d'édition et qui avait pour titre Setting Standards for Executive Performance. Le matériel de la présente étude provient d'un comité de recherche formé des représentants d'un grand nombre de compagnies; ce comité était sous la direction de l'auteur. Celui-ci a divisé scn ouvrage en six parties.

Dans la première partie, il nous démontre les résultats qui ont été atteints dons quelques compagnies par l'emploi d'objectifs et de standards de performance dans le "Management Process". Ces résultats semblent se concentrer sur le fait que la coordinction des efforts et l'unité dans l'entreprise s'améliorent lorsqu'il s'agit d'atteindre un: but déterminé.

L'cuteur poursuit dans la seconde partie en touchant du doigt le fait de considérer l'entreprise comme un système. Puis, il établit la similitude qui existe entre lés objectifs et les standards; si ceux-ci sont réalisables et spécifiques, de même qu'en relation ovec le budget et les plans de profit de l'entreprise, ils sont par contre dissemblables sur quelques points; ainsi les objectifs sont orientés vers les résultats, tandis que les standards sont orientés vers le contrôle.

L'établissement d'un programme pour chaque entreprise fait le sujet de la troisième partie. L'auteur veut nous démontrer la raison d'être d'une compagnie qui forme en quelque sorte sa charpente, ce qui la différenciero d'une autre compagnie. Ainsi, le programme d'une aciérie ne pourra être le même que celui d'une compagnie chimique, leur raison d'être n'étant pas semblcble. Mais chaque compagnie voudra avoir 
des résultats clés qui serviront par la suite à mesurer ses progrès. D'où l'établissement de différents programmes ayant à peu près les mêmes buts, soit le meilleur service possible qui opportera le meilleur profit possible tout en coordonnant les efforts des employés vers le même but, c'est-à-dire le succès de l'entreprise.

Dans la quatrième partie, l'auteur traite des différents programmes qui ont été tracés par environ 23 compagnies afin que les buts généraux de chacune de ces compognies soient en accord avec les buts de chaque employé. On y examine principalement les difficultés rencontrées, le manque de participation et de motivation de la part des employés, la manière avec laquelle les compagnies ont établi des standards ainsi que le nombre d'objectifs qui doivent être etublis pour un trovail donné.

L'outeur, dans la cinquième partie, revient un peu sur ce qui a été dit dans le chapitre 2, mais il fait ceci dans le dessein d'approfondir davantage l'importance qu'il $y$ a de ne pas séparer les objectifs et les standards de performance en ce qui a trait ò certaines activités qui sont d'une importance primordiale pour la direction, telles que le budget, les plans d'organisation et les descriptions de tâches, ainsi que l'évoluation de la performance et le développement de la main-d'oeuvre.

Pour conclure, l'auteur nous parle des aspects du futur en ce qui regarde l'entreprise. II voit celle-ci comme un système dynamique dans lequel les décisions programmiées et l'emploi des standards de performance se multiplieront et dans lequel le processus d'information sera de beaucoup amélioré

\section{Paul BELANGER}

\section{The Selection of Trainees Under MDTA,}

Institute of Management and Labor Relations, Rutgers - The State University, New Brunswick, New Jersey, 124 pages.

Cet ouvrage a pour objet l'examen du processus de sélection pour les travailleurs susceptibles de suivre des cours de formation professionnelle et la délimitation des caractéristiques des personnes choisies ou recalées et de celles qui n'ont jamais fait application pour suivre de tels cours.

Le présent rapport est le premier d'une série de deux. Le second permettro le développement de techniques spéciales de forma- tion professionelle pour les personnes qui ne sont pas capables de rencontrer les exigences sélectives, c'est-à-dire les personnes qui combinent une formation éducative inadéquate, de faibles aptitudes et une basse motivation.

Quant au contenu proprement dit du projet lui-même, disons qu'il se divise en trois sections. Dans une première section, les sujets qui y sont étudiés sont successivement la position du problème lui-même, le contexte où se situe l'étude, la population qui est l'objet de l'analyse, la classification de cette même population en fonction des règlements du MDTA, les échantillons choisis et finalement le plan du rapport.

La deuxième section, de son côté, concerne les caractéristiques des candidats qui se présentent aux progrommes du MDTA. On retrouve, par conséquent, des caractéristiques démographiques, des caractéristiques psychologiques et enfin des caractéristiques relatives au stotut occupationnel des travailleurs.

Pour terminer, le rapport retrace l'expérience préalable des travailleurs et élabore également sur leur expérience postérieure à leur période de formation professionnelle. II ne faudrait pas minimiser non plus l'importance que nous devons accorder à la description quelque peu sommaire du nombre et des divers types de cours organisés à partir des mesures gouvernementales du MDTA.

Si nous avions une appréciation à donner sur ce rapport, nous dirions qu'il est en mesure d'intéresser au plus haut point les responsables de la préparation et de la structuration des cours de formation professionnelle pour les travailleurs. Le problème de la sélection des candidats aux cours de formation professionnelle n'est pas encore résolu. Nous en sommes à la période d'essais et de tentatives. D'une part, il y a l'impératif d'un résultat positif toujours plus grand à atteindre et d'autre part, il y a le nombre passablement imposant de personnes rejetées en raison souvent de critères plutôt discutables. En fait, c'est le moment d'une prise de conscience et d'line remise en question des critères actuels qui devraient conduire à plus ou moins longue échéance à la reformulation ou au remplacement des critères actuels.

\section{Paul BOUCHARD}

L'importance croissante du secteur des services dans les pays membres de I'OCDE, por Maurice Lengellé, Organisation de Coopération et de Développement Economique, Paris, 1966, 144 pages. 